\title{
Formación continua en los profesionales: importancia de desarrollar las competencias investigativas en los docentes para el fortalecimiento de la educación universitaria
}

\section{Continuous training in professionals: importance of the developing research competences in teachers for the strengthening of the university education}

MORENO, Carola E. ${ }^{1}$

Resumen

La universidad propicia el encuentro e intercambio de saberes para solucionar las problemáticas profesionales y sociales. El objetivo de esta investigación fue Justificar la relevancia del desarrollo de las competencias investigativas en los docentes para el fortalecimiento de la educación universitaria. Se realizó la revisión de la literatura científica de trabajos de investigaciones y artículos de revistas especializadas, utilizando fichas sincréticas en la sistematización de información. Se concluye que, el desarrollo de las competencias investigativas en docentes universitarios es relevante.

Palabras clave: competencia, formación profesional continua, competencias investigativas.

\begin{abstract}
The university encourages the meeting and exchange of knowledge to solve professional and social problems. The objective of this research was to justify the relevance of the development of the research competencies in teachers for the strengthening of the university education. A review of the scientific literature of research papers and articles from specialized journals was carried out, using syncretic cards in the systematization of information. It is concluded that the development of research competencies in university teachers is relevant.
\end{abstract}

Keywords: competence, continuous professional training, research competencies.

\section{Introducción}

Dando respuesta a los cambios acelerados que genera la globalización, los avances tecnológicos y las circunstancias actuales que se afrontan en la educación superior, es necesario fomentar el desarrollo de las competencias investigativas en los docentes, como parte de la formación profesional continua, para el fortalecimiento de la educación universitaria. En Perú, como en muchos otros países, el sistema educativo persigue un enfoque por competencias; en la Ley del profesorado $\mathrm{N}^{\circ} 24029$, con las modificatorias de la Ley $\mathrm{N}^{\circ}$ 25212, establece los lineamientos de la mejora profesional continua. A través de la Ley universitaria $\mathrm{N}^{\circ} 30220$, señala; la investigación y el desarrollo de competencias investigativas en los docentes y estudiantes, es una función obligatoria y prioritaria de la universidad. Así mismo la Ley Universitaria $\mathrm{N}^{\circ} 23733$, considera importante

${ }^{1}$ Docente de Educación Básica Regular y Docente Universitaria. Perú. masterteacingcmv@gmail.com 
la función universitaria, puesto que, es un aspecto considerado en el proceso de evaluación para la certificación, licenciamiento y acreditación. (Ministerio de Educación - MINEDU, 2018, 2020a, 2020b).

La competencia investigativa es relevante en la formación profesional, pues responde a las necesidades específicas de formación y posterior desempeño de cada profesional. A medida que avanza el proceso de investigación, las competencias investigativas incrementan. La educación superior es responsable del despliegue de competencias profesionales e investigativas, su papel básico es capacitar profesionales, científicos e investigadores de alto nivel, reconsiderando los objetivos en la sociedad del conocimiento a través de la investigación científica; y redefiniendo el papel del profesor como investigador (Banderas Martínez et al., 2018; D’olivares Durán et al., 2019; Díaz de Salas, 2020; Guamán Gómez, Verónica Jacqueline, Herrera Martínez, Lázara, \& Espinoza Freire, 2020).

A través de la investigación, el docente muestra responsabilidad social, lo que permite expresar orgánicamente las habilidades básicas y complementarias de gestión del conocimiento: seleccionar y clasificar información, realizar procesamientos de información de manera critica, generar nuevos conocimientos y nuevos resultados y, finalmente comunicarlos y socializarlos (Banderas Martínez et al., 2018; Guamán Gómez et a., 2020; Lunamenecio, 2020; Quezada Castro et al., 2020; Reiban et al., 2017; Turpo-Gebera et al., 2020; Vallejo, 2020).

Mendivel (2017) y Núñez (2019) sostienen que los docentes aún carecen de competencias investigativas, al momento de realizar producciones académicas y asesoramiento. Es así que en la formación continua de los profesionales cobra vital importancia el desarrollo de las competencias investigativas de los docentes -; en donde se integran los siguientes aspectos; el cognitivo, la puesta en escena de habilidades y destrezas del saber ser, saber hacer y el saber transferir. El desarrollo de competencias investigativas, implica usar los conocimientos de forma apropiada, afianzando las capacidades observacionales, el cuestionamiento, la argumentación, la sistematización, gestionando los conocimientos, sobre la base del interés y la motivación hacia la investigación y la realización personal y profesional.

En concordancia con todo lo anteriormente expuesto, se plantea el siguiente objetivo general: justificar la relevancia del desarrollo de las competencias investigativas en los docentes para el fortalecimiento de la educación universitaria. Los objetivos específicos son: (a) conceptualizar las variables de estudio: formación continua y competencia investigativa, (b) describir las competencias investigativas del docente universitario, (c) explicar el rol de la universidad en el desarrollo de las competencias investigativas. Se considera relevante el desarrollo de las competencias investigativas en los docentes -, como parte de la formación profesional continua; donde se integra un conjunto de saberes, que potencian su desempeño en concordancia con el nivel del desarrollo de esta competencia, que se revela como una necesidad actual en el entorno de la educación superior universitaria tanto nacional como internacional.

\section{Metodología}

La metodología utilizada en el presente trabajo académico fue la revisión de la literatura científica de trabajos de investigaciones y artículos de revistas especializadas relacionados con el tema de estudio, lo cual representó la base teórica. Dicha revisión ha permitido reflexionar sobre la importancia del desarrollo de las competencias investigativas en la labor del docente universitario. Esta metodología puede aplicarse a diversos temas de investigación, da respuestas a diversas interrogantes, lo que permite determinar cuán importante es la investigación. Al ser un estudio exploratorio, intenta precisar las características, perfiles importantes de la unidad de análisis. La investigación de revisión de la literatura científica, tiene como propósito recopilar información y enunciar teorías que sustenten el estudio y proceso (Cabezas et al., 2018; Delgado et al., 2019). 
En la revisión de la literatura científica el primer paso del investigador es recopilar información de libros, revistas científicas, sitios web e información relacionada al tema a estudiar. Los trabajos elegidos son aquellos que desarrollan temas de interés y que constituyen contribuciones culturales o científicas. -. (Hernández Sampieri et al., 2014; Gómez-Luna et al., 2014; Véliz \& Torres, 2017; Baena \& Baena, 2017, p. 46-66). Algunas características de la revisión de la literatura científica son: es sintética, resalta los documentos consultados que más ayudan a comprender el problema de investigación, presenta los conocimientos de forma crítica, las fuentes deben ser actuales, valora los estudios que influyen en el desarrollo disciplinar, ya que tiene especial relevancia en el ámbito académico.

\subsection{Sistematización de la información}

La sistematización de la información es una etapa importante en cualquier proyecto de investigación, incluye la organización sistemática de los documentos encontrados. Barbosa-Chacón (2015), señala que, la sistematización de la información responde a las siguientes interrogantes: el ¿qué?, al definirse a partir de un término asociado a la experiencia que se sistematiza; el ¿cómo?, alude a los principios metodológicos; el ¿para qué?, se detalla aquello en lo que la se contribuye. Expósito y Gonzales (2017), sostienen que, en el proceso de sistematización hay una intencionalidad transformadora, ya que constituye un resultado científico, el mismo que conlleva a la transformación que se propone el investigador.

\subsection{Gestión de la información}

Los programas de gestión para organizar las fuentes de referencia se han convertido en herramientas muy útiles en el desarrollo de la investigación científica. El gestor de fuentes de referencia es un programa informático que se utiliza para la recopilación, almacenamiento y gestión de dichas fuentes - en los trabajos científicos. Además, estos evitan al investigador memorizar manuales, redactar las citas una a una; ahorra tiempo y esfuerzo; siendo Mendeley uno de los gestores más utilizados (López Carreño, 2015; Gallegos et al., 2017; De Cos GonzalezTaladriz \& Brizo-Montiano, 2017)

El gestor de fuentes de referencia utilizado en la sistematización del presente trabajo de investigación fue el Mendeley; al respecto De Cos Gonzalez-Taladriz \& Brizo-Montiano (2017), sostienen que, es un gestor de información que facilita la captura y organización de las fuentes referencia, los trabajos con documentos y las citas; además brinda múltiples formatos el estilo de redacción científica. Mendeley es una red social que incorpora distintas funcionalidades, permite crear equipos de trabajo y compartir las referencias con usuarios de una red social online. Además, se siguió los lineamientos de redacción académica proporcionados por la Guía de Elaboración de Trabajos de Investigación de la Universidad César Vallejo (UCV, 2020), y las Normas de American Psychological Association (Guía Normas APA 7a edición, 2020).

\subsection{Técnica e instrumento de investigación}

El presente trabajo de investigación utilizó la técnica del fichaje; este sirve para registrar, seleccionar y sistematizar información específica de documentos consultados. Como técnica de estudio e investigación, facilita recopilar, seleccionar, organizar y conservar de forma ordenada los datos del trabajo de investigación. El instrumento utilizado para complementar la técnica fue la ficha sincrética, un instrumento que permite al investigador la organización de la información; con la finalidad de concatenar las ideas respecto al tema estudiado. Acorde con Castro (2020), algunas de las fichas de trabajo y contenido son fichas de citas textuales o citas directas, fichas de resumen, fichas de traducción o paráfrasis (Andean University, 2018; Castro Taks, 2020; De los Santos Alejos, 2015). 


\section{Resultados}

\subsection{Formación continua}

La formación continua es una herramienta que promueve a que las personas se preocupen de seguir preparados y de buscar el desarrollo profesional para mejorar su competitividad en el lugar y/o plaza de trabajo. La formación profesional es; inicial o continua. La formación inicial, está referida a la previa preparación del ejercicio profesional, por ende, están inmersos los cursos de licenciatura; y la formación continua, referida al momento en el cual el docente ya ejerce la profesión, y es reconocido algunas veces con reconocimientos oficializados como la maestría o el doctorado. (Alanís, 2018; Díaz, et al., 2020).

Los conceptos y la terminología referente a formación continua, se refieren a las características de identidad e imagen profesional. A partir de las reformas educativas y curriculares de la década de 1990, la capacitación comenzó a crecer en gran escala y recibió mayor atención en la agenda del gobierno. En la mayoría de países, las actividades de capacitación son realizadas por las distintas áreas o direcciones responsables de educación. En el sector estatal se lleva a cabo capacitaciones en forma continua, en tanto en el sector privado a veces son realizados por las empresas editoriales, como estrategia de ventas para sus textos, otras veces en centros privados de formación - docente o universidades (Vezub,2019).

La formación continua se relaciona con la capacidad, la mejora personal, el autoconocimiento, la autoestima, el autocontrol o la motivación, la creatividad, el cambio o la capacidad de tomar decisiones; por otro lado, mejora también las relaciones con los demás, desarrollando la empatía, la capacidad de establecer una comunicación asertiva, la capacidad de trabajar en equipo, la resolución de conflictos y análisis de las necesidades sociales. Tal como Maslow en su teoría motivacional señala como base fundamental las necesidades fisiológicas y la de seguridad, más alto que la de filiación y en la cúspide se encuentra la autorrealización, en la cual se incluye la formación continua. Al estar bien preparado y actualizado, el profesional no teme al despido, está seguro de sí mismo, autorrealizado, con un sentimiento de trascendencia y progreso personal. (Cegos, 2020; Valdés \& Gutiérrez, 2018)

La formación continua se da en comunidades de aprendizaje, donde se reúne un grupo de personas, con el propósito de participar activamente en la búsqueda de soluciones a situaciones relacionados con la sociedad y el entorno laboral. Está dirigida al desarrollo de habilidades profesionales complejas, la capacidad macro de enseñanza es crucial, esta permite el logro de un mejor desempeño docente. Así el desarrollo de las competencias docentes, se ve favorecido, pues mejora el manejo de estrategias en el campo pedagógico y disciplinar, permite reflexionar sobre la práctica profesional complementado con la innovación, investigación y evaluación basada en una teoría crítica a la vanguardia de las demandas profesionales a nivel mundial. Asegurando así la empleabilidad y reconocimiento futuro (Albareda-Tiana et al., 2018; Arandia et al., 2019; Dios et al., 2018; Matute \& Pereira, 2020; Rodríguez et al., 2019).

En la mayoría de países, las actividades de capacitación son realizadas por las distintas áreas o direcciones responsables de educación, no existe una institución u organización de dirección especializada. Además, con la propuesta de carácter nacional se ha llevado a cabo capacitaciones en forma continua en el sector privado (Vezub, 2019).

\subsection{Competencias investigativas}

El concepto de competencia ha evolucionado históricamente, siendo definida desde las diversas ciencias y perspectivas, incluidas la psicología, la lingüística, la sociología y la pedagogía. La palabra competencia tiene gran realce porque va ligada con la parte cognitiva, comunicativa y comportamental, pues permite actuar y desenvolverse de manera pertinente en la vida diaria. En el ámbito de la competitividad, es la capacidad para 
resolver un problema o realizar una actividad específica eficientemente. Comprendida desde la incumbencia, alude a la capacidad de relacionar conceptos adquiridos con la realidad; involucrarse con responsabilidad y ética en la sociedad, con un desarrollo personal holístico, con un proyecto de vida basada en la ética y cooperación, para enfrentar los desafíos actuales y futuros; con una buena organización ambiental, social, cultural y de desarrollo (Rodríguez et al., 2019; Ramos y Silva, 2019).

El MINEDU (2020), en la resolución viceministerial $N^{\circ} 033-2020$, indica que competencia es la aptitud que posee una persona para combinar todas las habilidades y capacidades con la finalidad de actuar de manera pertinente, oportuna y ética en una situación dada para lograr un propósito específico. Por su parte Ramos y Silva (2019) señalan que el término competencia tiene valor semántico en distintas áreas del saber; en la biología, el deporte, la economía, la lingüística, etc. En el contexto pedagógico está vinculado al proceso de formación y las formas en que se modifican las estructuras en la mente, para lograr una clara visión sobre la realidad, y así se logra mejoras en el desempeño profesional.

En muchos casos, los cursos de formación para profesores universitarios no especifican los principales fines de acción, pero se especifica en el plan de gestión docente del curso académico involucrado, por tal motivo han creado una cuarta categoría denominada formación general. Se refiere a los cursos dirigidos al personal docente y administrativo, tanto en formación inicial y la formación continua. Normalmente, se clasifican en 12 cursos por temáticas, estrechamente vinculadas en la tarea docente: docencia general, planificación, metodología, evaluación, tutoría, Tecnologías de la Información y Comunicación (TICs), Igualdad, ética y compromiso social, investigación, idiomas, inclusión y creatividad. (Alanís, 2018).

La Organización de las Naciones Unidas para la Educación, la Ciencia y la Cultura UNESCO (2020), enfatiza que la docencia está vinculada a la investigación científica y la generación de conocimiento. Por su parte, el papel de la extensión universitaria es buscar soluciones a los problemas y atender las necesidades de contexto, a través de acciones específicas. En la formación docente se considera: (a) Seguimiento de los instrumentos normativos internacionales sobre enseñanza; (b) Apoyar a los Estados Miembros en la formulación y revisión de políticas y estrategias relacionadas con los docentes; (c) Desarrollo de las capacidades para mejorar la calidad de la enseñanza; (d) En la Agenda de Educación 2030 para la implementación y monitoreo de la mejora del conocimiento y la base de hechos en las metas de los maestros, y (e) Promover e intercambiar conocimientos para promover un proceso de enseñanza y aprendizaje de alta calidad. (p. 1-2).

\subsection{El rol de la universidad en el desarrollo de competencias investigativas}

El MINEDU (2018), en la Ley Universitaria 30220, art. Nº3 define a la universidad como; comunidad académica universitaria articulada a la utilización de la ciencia y tecnología para generar innovación haciendo uso de la práctica investigativa, siendo los estudiantes protagonistas de emprender proyectos investigativos para brindar solución a los problemas. Esta ley señala cuatro misiones de la universidad: (a) misión investigativa; la misión más importante en la práctica de docentes y estudiantes, (b) misión económica; utilizar los recursos y presupuestos de manera transparente. (c) misión social; hacer realidad la ejecución de los servicios de extensión

y programas de bienestar universitario, (d) misión ambiental; emprender una enseñanza que propicie el respeto a los valores ambientales, la interacción de aprendizaje, innovación y generación de conocimientos a través de la indagación, análisis e interpretación.

Los principios, fines y funciones de la Universidad, contemplados en la Ley Universitaria 30220 enfatizan la importancia del desarrollo de las competencias investigativas, tanto en docentes como en estudiantes, es así que, el gobierno en los últimos años, con el impulso del Consejo Nacional de Ciencia, Tecnología e Innovación Tecnológica (CONCYTEC), el Ministerio de la Producción y la propia universidad, busca el fortalecimiento del sistema educativo, buscando garantizar la calidad de las universidades. Su proceso incluye licenciamiento y 
certificación universitaria, estos procesos dan prioridad a la producción académica, implementación de laboratorio, etc. Como se indica en el último Informe Bienal de la Superintendencia Nacional de Educación Superior (SUNEDU, 2017).

La investigación es una parte central de las universidades, y son ellas quienes contribuyen a través de la educación en: fortalecer el trabajo de investigación y mejorar el conocimiento y la capacidad de los científicos, expertos y futuros líderes. En muchos países, incluido el nuestro, las regulaciones universitarias requieren que los estudiantes obtengan su título profesional con la preparación y sustentación de una tesis; sin embargo, no todos los estudiantes muestran las competencias necesarias de investigación, ya que no han recibido las pautas necesarias para ello. En esos momentos, se presentan algunas de las limitaciones las cuales obstaculizan o frustran el desarrollo de su investigación. En muchos casos, esta frustración es generalizada y evita por ejemplo que nuevos investigadores ingresen a la escuela de posgrado. (UNESCO, 2020).

La necesidad de una conexión permanente entre la enseñanza universitaria y la investigación ha sido ampliamente reconocida, lo cual es un requisito para la mejora de la calidad educativa. Está asociada con la actualización y mejora del personal docente, que se especifica en la transferencia permanente de la experiencia y los resultados del proceso de investigación a la enseñanza-aprendizaje. La mayoría de las investigaciones están dirigidas a trabajar con los estudiantes en el proceso de enseñanza; aunque algunos de ellos hablan sobre la formación continua que se deben dar a los maestros. Las estrategias institucionales también deben estar presentes, como brindar incentivos, tener el compromiso para financiar los proyectos innovadores, brindar recursos para apoyar el desarrollo continuo de los docentes; por ejemplo, dar conferencias, seminarios. (Cient et al., 2018; Feixas \& Zellweger, 2019)

Los programas de formación docente respecto a sus contenidos, se sitúan en los siguientes ámbitos:

- Ámbito pedagógico; comprendido por temas dispersos, incluidos los del campo didáctico y del epistemológico en lo que respecta al campo educativo.

- Ámbito investigativo; orientado a la formación correspondiente a la metodología investigativa, y al desarrollo de las competencias de lectura y escritura para realizar producciones intelectuales.

- Ámbito tecnológico; consiste en la apropiación del conocimiento y manejo básico de las herramientas informáticas, y el uso de plataformas e-learning.

- Ámbito humanístico; corresponden a temas desde la identidad institucional, hasta los conocimientos de legislación referentes a la educación superior; las que pasan por; ética general, deontología y responsabilidad social docente.

- Ámbito de internacionalización; estos programas de formación se orientan a favorecer la movilidad de estudiantes y docentes universitarios, incluye también la enseñanza del idioma inglés tanto en los contenidos como en la comprensión lectora en la lengua extranjera.

- Ámbito disciplinar; relacionado a la disciplina o ciencia correspondiente al docente, desde la titulación universitaria hasta el desempeño laboral, en este último ámbito, la formación se da por cuenta e iniciativa del propio docente, esto se da a través de programas educativos formales en el nivel de postgrado. (Lopézde Parra et al., 2017).

Alanís (2018) sostiene que pese a su importancia estratégica, la formación para docentes universitarios -, ha sido mínimamente investigada. Las universidades realizan: cursos, foros, talleres, conferencias, etc., dirigidos a la formación continua de sus docentes; además de tener en cuenta - los objetivos específicos, se exige cumplir con ciertos elementos del sistema de formación profesional como: los objetivos, los materiales, los participantes, los 
programas, los espacios, las coordinaciones, el personal facilitador, etc., en adición se incluyen también los cursos de licenciatura y posgrado. -

Los programas de posgrado, esperan que los estudiantes participen plenamente en la revisión e interpretación investigativa, pues asumen que se trata de una habilidad desarrollada a nivel de pregrado. (Hubbard \& Dunbar, 2017). Aún existen muchas falencias respecto al desarrollo de trabajos de investigación en el pregrado, hay una necesidad urgente de aplicar un currículo que conlleve al desarrollo de habilidades de investigación científica y una actitud positiva hacia ella, pues son aplicables siempre y cuando las percepciones y habilidades docentes lo permitan. (Hüseyin \& Tarhan, 2015). El enfoque holístico de la formación en competencias científicas se basa en tres pilares: (i) un plan de estudios científicos básico, (ii) proyectos de investigación intracurriculares y (iii) programas de investigación especiales para estudiantes con un alto nivel de interés en la investigación. (Ratte et al., 2018)

Basado en la formación académica y el conocimiento adquirido de la asignatura, el docente debe proponer ideas apropiadas para la transferencia de conocimientos entre los distintos actores educativos e integrar los diferentes procedimientos y contenidos que componen la experiencia curricular. Pero, frente al proceso de transformación, algunos docentes universitarios, se resisten al cambio de paradigma, y a las nuevas políticas educativas, incluida la investigación; es necesaria la implementación de un plan de actualización continua para todos los docentes, para afianzar el desarrollo de las competencias investigativas, mejorando así su práctica docente. Los docentes universitarios deben tener apertura al cambio, con una visión de enseñanza transformadora, lo que conlleva a mejorar su propia reputación y la de su institución donde labora. (Feixas \& Zellweger, 2019; Flores et al., 2020).

En este sentido Flores, et al. (2020) manifiesta que los docentes deben cultivar una cultura investigativa entre los estudiantes de acuerdo con las siguientes funciones: Cultivar la curiosidad y el espíritu de descubrimiento y busqueda permanentemente de lo desconocido; Guiar proyectos de investigación utilizando métodos adecuados para las ciencias naturales y sociales; Cumplir con la ética, promoviendo el debate, intercambio de ideas y el respeto por las prácticas de investigación; Apoyar a los estudiantes en materiales, suministros, equipos, herramientas, laboratorios, investigación, etc.

\subsection{Etapas y dimensiones de las competencias investigativas}

Dentro de la formación profesional, es importante el desarrollo las competencias investigativas, se pueden ejecutar y verificar las acciones dentro del mismo desarrollo profesional. El conocimiento proporciona un significado global para la investigación, y brinda las estrategias e integridad científica, es decir, el respeto a la moral y al rigor científico. Del mismo modo, también permite adquirir conocimiento organizacional y sistemático, y expandir el conocimiento a través de la difusión de informes académicos con los resultados científicos. Para los participantes en investigación, la experimentación y la observación son procedimientos importantes de investigación, pues requieren de una actitud crítica compatible con la formación continua (Turpo, et al., 2020). Ponce et al. (2020), reconoce cuatro dimensiones de las competencias investigativas: (a) la epistemológica. (b) la metodológica. (c) la técnica, y (e) la social.

\subsection{Habilidades de las competencias investigativas}

Böttcher \& Thiel (2018) describen y resumen las características comunes de la práctica de investigación; en un conjunto de habilidades requeridas para llevar a cabo la investigación en varias disciplinas académicas:

- Revisar sistemáticamente hallazgos relevantes anteriores sobre un tema, evaluar la calidad de publicaciones relevantes anteriores, identificar las necesidades de investigación (habilidades para revisar el estado de la investigación). 
- Investigar la pregunta de investigación de una manera metodológicamente controlada y sistemática, operacionalizando sistemáticamente aspectos relevantes del objeto de investigación, y analizando sistemáticamente la información recopilada (habilidades metodológicas).

- Reflexionar sobre las limitaciones, así como las implicaciones teóricas, éticas y prácticas (habilidades que reflejan interés respecto a los resultados de una investigación).

- Presentar el o los resultados del estudio de investigación (oral y escrita) de acuerdo con los estándares profesionales, de manera que el enfoque metodológico sea transparente para los miembros de la comunidad científica (habilidades de comunicación).

\subsection{Importancia de las competencias investigativas}

En los conceptos tradicionales de enseñanza e investigación, ya existía un desafío para conseguir el equilibrio entre el conocimiento existente y el nuevo conocimiento: la enseñanza puede extender la base disciplinaria de la investigación y el desarrollo del proceso de enseñanza-aprendizaje. Pero hoy, se requiere resolver problemas adicionales a la educación tales como; el medio ambiental, el cambio climático, los recursos naturales y la energía; la variación demográfica, los flujos migratorios, la salud, y la digitalización. Hay interacciones entre los campos profesionales, y búsqueda de soluciones interdisciplinarias para lograr la viabilidad futura de la sociedad; es necesario encontrar métodos alternativos que no interrumpan el equilibrio; en este sentido, las universidades deben desarrollar en los graduados las habilidades para resolver los problemas de manera responsable (Feixas \& Zellweger, 2019).

Las competencias investigativas ayudan a la profundización de la formación científica e investigativa, enmarcado en el enfoque por competencias y una educación de calidad, para el aprendizaje útil para la vida y una formación en valores. El desafío consiste en reconocer el papel de las universidades en una sociedad globalizada, en la que es necesario redefinir el papel de los docentes como investigadores $y$, al mismo tiempo, desarrollar las habilidades en los estudiantes para que sean capaces de generar y gestionar el conocimiento en el entorno laboral. Estudiar, criticar, innovar, inventar y creer firmemente que la forma de romper la dependencia, es la investigación. (Contreras \& Maluk, 2017; Reiban et al., 2017; Rodríguez et al., 2019).

Buendía et al. (2017) señala que, quienes pueden abordar desde la experiencia de su misma práctica a través de la investigación, son los docentes en formación, puesto que es una gran oportunidad para entrar de lleno al conocimiento y a la vez encontrar soluciones de manera sistemática a los diversos problemas que se presentan al estar inmersos en el campo educativo. Respecto a la importancia del desarrollo de las competencias investigativas, señalan los siguientes atributos clave:

- Comprensión del significado, importancia e implicancia de las investigaciones en el campo educativo y en la praxis pedagógica del docente.

- Competencias propositivas, cuyo fin es proponer solución a los problemas.

- Competencias tecnológicas, relacionada con la técnica de selección y recojo de datos y el uso del software para analizar los datos y exponer resultados.

- Competencias interpersonales, relación armónica con los sujetos implicados.

- Competencias cognitivas, conocer, analizar, comparar, evaluar, interpretar teorías, métodos, etc.

- Competencias procedimentales, destreza y el manejo de las técnicas al desarrollar la investigación.

- Competencias analíticas, comprensión profunda del tema. 
- Competencias comunicativas, para generar y para difundir el conocimiento como resultado de la investigación. (p. 184-185).

Las competencias investigativas contribuyen con el fortalecimiento del desempeño de la educación superior, orienta al estudiante, facilita la adquisición de saberes para dar alternativas de solución integral, haciendo uso de la tecnología y los procesos de educación productiva. Es decir, las competencias investigativas están compuestas por un conjunto de herramientas dirigidas a la mejora de los aprendizajes. Se manifiesta en la producción de conocimientos, uso adecuado del método científico y de la divulgación de tal conocimiento como ciencia. Es necesario impulsar planes de investigación, como la búsqueda especializada de información en las bases de datos, los conceptos de lectura y escritura con sentido crítico y los métodos de trabajo universitarios; para facilitar el proceso de un buen estudio investigativo. (Gómez-Escorcha et al., 2019; Rodríguez et al., 2019).

Tinoco-Cuenca et al. (2020) señala la importancia de continuar fortaleciendo la cultura de investigación relacionada con la capacitación. Los graduados con habilidades de investigación pueden beneficiar a las instituciones educativas y las sociedades de investigación. Además, se recomienda fortalecer y mejorar el sentido de pertenencia a las universidades, para que los graduados estén orgullosos de su alma mater, y estar involucrados en ella, participando activamente en los programas curriculares de actualización y en los proyectos de investigación para fortalecer y reconocer las instituciones superiores.

\section{Conclusiones}

La formación continua se relaciona con la capacidad, la mejora personal, el autoconocimiento, la autoestima, el autocontrol o la motivación, la creatividad, el cambio; también, mejora las relaciones interpersonales, desarrollando la empatía, la comunicación asertiva, la resolución de conflictos, el trabajo en equipo y el análisis de las necesidades sociales.

Las competencias investigativas contribuyen con el fortalecimiento del desempeño de la educación superior, orienta al estudiante, facilita la adquisición de saberes para dar alternativas de solución integral, haciendo uso de la tecnología y los procesos de educación productiva. La investigación es una parte central de las universidades, y son ellas quienes contribuyen a través de la educación en: fortalecer el trabajo de investigación y mejorar el conocimiento y la capacidad de los científicos, expertos y futuros líderes.

Los docentes universitarios deben tener apertura al cambio, con una visión de enseñanza transformadora, lo que conlleva a mejorar su propia reputación y la de su institución donde labora. Es importante reconocer el papel de las universidades en una sociedad globalizada, en la que es necesario redefinir el papel de los docentes como investigadores y, al mismo tiempo, desarrollar las habilidades investigativas de los estudiantes para que sean capaces de generar y gestionar el conocimiento en el entorno laboral.

Es relevante el desarrollo de las competencias investigativas en los docentes para el fortalecimiento de la educación universitaria; pues ayudan a la profundización de la formación científica e investigativa, enmarcado en el enfoque por competencias y una educación de calidad, para el aprendizaje útil para la vida y una formación en valores. El conocimiento proporciona un significado global para la investigación, y brinda las estrategias e integridad científica, es decir, el respeto a la moral y el rigor científico.

\section{Referencias bibliográficas}

Alanís Jiménez, J. F. (2018). Consideraciones para la formación continua de personal docente universitario. Revista Educación, 228-249. Recuperado de: https://doi.org/10.15517/revedu.v42i2.25123 
Albareda-Tiana, S., Vidal-Raméntol, S., Pujol-Valls, M., \& Fernández-Morilla, M. (2018). Holistic Approaches to Develop Sustainability and Research Competencies in Pre-Service Teacher Training. Sustainability, 10(10), 3698. Recuperado de: https://doi.org/10.3390/su10103698

ANDEAN UNIVERSITY. (2018). Técnicas del Fichaje 2018 -II.doc - Universidad Andina Del Cusco Tlu00c9cnicas de estudio y de Investilu00d3n I Actividad inicial de recojo de saberes/Course Hero. Recuperado de: https://www.coursehero.com/file/42286873/TECNICAS-DEL-FICHAJE-2018-IIdoc/

Baena, G., \& Baena, P. (2017). Metodología de la investigación Grupo Editorial Patria Sistema de aprendizaje en línea Metodología de la investigación. Recuperado de: www.editorialpatria.com.mxwww.sali.org.mx

Banderas Martínez, C., Olivares, G. C., Estela, M., \& Castro, M. (2018). Perspectivas docentes sobre la formación de competencias investigativas en relación con los programas de asignatura. Sincronía, ISSN-e 1562-384X, No. 74 (Julio-Diciembre 2018), 2018, págs. 589-616, 74, 589-616. Recuperado de: https://doi.org/10.32870/sincronia.axxii.n74.29b18

Barbosa-Chacón, Jorge Winston; Jarbosa Herrera, uan Carlos; Rodríguez Villabona, M. (2015). Concepto, enfoque y justificación de la sistematización de experiencias educativas: Una mirada "desde" y "para" el contexto de la formación universitaria. Recuperado de:

http://www.scielo.org.mx/scielo.php?script=sci_arttext\&pid=S0185-26982015000300008

Böttcher, F., \& Thiel, F. (2018). Evaluating research-oriented teaching: a new instrument to assess university students' research competences. Higher Education, 75(1), 91-110. Recuperado de:

https://doi.org/10.1007/s10734-017-0128-y

Buendía Arias, X. P., Zambrano Castillo, L. C., \& Insuasty, E. A. (2017). El desarrollo de competencias investigativas de los docentes en formación en el contexto de la práctica pedagógica. Revista Folios, 47. Recuperado de: https://doi.org/10.17227/folios.47-7405

Castro Taks, A. (2020). ELABORACIÓN DE FICHAS ¿Qué son las fichas? Recuperado de: https://investigar1.files.wordpress.com/2010/05/elaboracic3b3n-de-fichas.pdf

CEGOS. (2020). La importancia de la formación continua para el profesional - Cegos. Recuperado de: https://www.cegosonlineuniversity.com/la-importancia-de-la-formacion-continua-para-el-profesional/

Contreras, F. A. G., \& Maluk Uriguen, S. A. (2017). A descriptive analysis of university administration in ecuador from the perspective of legislative change. Revista Electronica de Investigacion Educativa, 19(2), 22-37. Recuperado de: https://doi.org/10.24320/redie.2017.19.2.866

D’olivares Durán, N., Liliana, C., \& Cifuentes, C. (2019). Competencias investigativas: inicio de formación de jóvenes investigadores en educación media. Humanismo. Soc, 7(1), 2019. Recuperado de: https://doi.org/10.22209/rhs.v7n1a01

Damián Cabezas Mejía, E., \& Andrade Naranjo Johana Torres Santamaría, D. (2018). Introducción a la metodología de la investigación científica. Recuperado de: www.repositorio.espe.edu.ec.

De Cos Gonzalez-Taladriz, Lucía \& Brizo-Montiano, S. M. (2017). Mendeley Guía de uso Grupo de Trabajo de Gestores Bibliográficos. Recuperado de: https://biblioteca.ucm.es/data/cont/docs/382-2018-01-23Manual Mendeley 3 ed. (Noviembre 2016)reducido.pdf

De los Santos Alejos, M. A. (2015). El fichaje como metodo de investigacion. Recuperado de: https://es.slideshare.net/MADLSA/el-fichaje-como-metodo-de-investigacion 
Delgado, et al. (2019). Competencias Investigativas del Docente Construidas durante la Formación Universitaria. Revista Scientific, 4(13), 200-220. Recuperado de:

https://doi.org/10.29394/scientific.issn.2542-2987.2019.4.13.10.200-220

Díaz de Salas, Y. del C. (2020). Mirada Fenomenológica desde las Competencias Investigativas en las Líneas de Investigación de la UNESR. Revista Scientific, 5(15), 269-287. Recuperado de: https://doi.org/10.29394/scientific.issn.2542-2987.2020.5.15.13.269-287

Dios, I., Calmaestra, J., \& Rodríguez-Hidalgo, A. J. (2018). Validación de la escala de competencias docentes organizacionales y didácticas para educadores. Revista Mexicana de Investigacion Educativa, 23(76), 281302. recuperado de: https://www.revistaespacios.com

Expósito Unday, Dámari ; Gonzales Valero, J. A. (2017). Sistematización de experiencias como método de investigación. Recuperado de: http://scielo.sld.cu/scielo.php?script=sci_arttext\&pid=S160889212017000200003

Feixas, M., \& Zellweger, F. (2019). Formación e innovación docente universitaria para una educación transformadora: la investigación e indagación reflexiva como requisito. El Guiniguada. Revista de investigaciones y experiencias en Ciencias de la Educación. Recuperado de: https://doi.org/10.20420/elguiniguada.2019.265

Flores Nessi, E. M., Loaiza Falcón, A. C., \& Rojas de Ricardo, G. N. (2020). Rol del docente investigador desde su práctica social. Revista Scientific, 5(15), 106-128. Recuperado de:

https://doi.org/10.29394/scientific.issn.2542-2987.2020.5.15.5.106-128

Gallegos, M. C., Peralta, C. A., \& Guerrero, W. M. (2017). Utilidad de los gestores bibliográficos en la organización de la información para fines investigativos. Formacion Universitaria, 10(5), 77-85. Recuperado de: https://doi.org/10.4067/S0718-50062017000500009

Gómez-Escorcha, J. A., Isea Araque, N., \& López Lara, V. de las M. (2019). Competencias investigativas y su influencia en la formulación de proyectos de investigación en la Universidad Valle del Momboy. Actualidades Pedagógicas, 1(73), 191-213. Recuperado de: https://doi.org/10.19052/ap.vol1.iss74.10

Gómez-Luna, E., Fernando-Navas, D., Aponte-Mayor, G., \& Betancourt-Buitrago, L. A. (2014). Metodología para la revisión bibliográfica y la gestión de información de temas científicos, a través de su estructuración y sistematización. DYNA (Colombia), 81(184), 158-163. Recuperado de: https://doi.org/10.15446/dyna.v81n184.37066

Goris, G. (2015). UTILIDAD Y TIPOS DE REVISIÓN BIBLIOGRÁFICA / Guirao Goris / Revista Ene de Enfermería. Recuperado de: http://ene-enfermeria.org/ojs/index.php/ENE/article/view/495/guirao

Guamán Gómez, Verónica Jacqueline, Herrera Martínez, Lázara, \& Espinoza Freire, E. E. (2020). Las competencias investigativas como imperativo para la formación de conocimientos en la universidad actual. Recuperado de: http://scielo.sld.cu/scielo.php?script=sci_arttext\&pid=S1990$86442020000100083 \& \operatorname{lng}=e s \& n r m=i s o \& \operatorname{lng}=e s$

Guía Normas APA $7^{a}$ edición. (2020). Recuperado de: https://normas-apa.org/

Hubbard, K. E., \& Dunbar, S. D. (2017). Perceptions of scientific research literature and strategies for reading papers depend on academic career stage. PLOS ONE, 12(12). Recuperado de: https://doi.org/10.1371/journal.pone.0189753 
Hüseyin Şahan, H., \& Tarhan, R. (2015). International Journal of Psychology and Educational Studies Scientific Research Competencies of Prospective Teachers and their Attitudes toward Scientific Research ARTICLE INFO ABSTRACT. International Journal of Psychology and Educational Studies, 2(3), 20-31. Recuperado de: https://doi.org/10.17220/ijpes.2015.03.003

Lopéz- de Parra, L., Polanco-Perdomo, V., \& Correa-Cruz, L. (2017). Mirada a las investigaciones sobre formación investigativa en la universidad latinoamericana: estado del arte 2010 a 2017. REVISTA DE INVESTIGACIÓN, DESARROLLO E INNOVACIÓN, 8(1), 77. Recuperado de: https://doi.org/10.19053/20278306.v8.n1.2017.7371

López Carreño, M. (2015). Análisis comparativo de los gestores bibliográficos sociales Zotero, Docear y Mendeley: características y prestaciones. Recuperado de: https://digitum.um.es/digitum/bitstream/10201/51054/1/Analisis comparativo de los gestores bibliograficos sociales Zotero, Docear y Mendeley caracteristicas y prestaciones..pdf

Luna-menecio, A. A. (2020). La construcción de competencias investigativas del docente de posgrado para lograr el desarrollo social sostenible. Recuperado de: https://www.revistaespacios.com

Matute, L. J., \& Pereira Rodríguez, Z. (2020). Práctica investigativa: Diferentes tiempos y visiones en la formación universitaria. Revista EDUCARE - UPEL-IPB - Segunda Nueva Etapa 2.0, 24(1), 303-319. Recuperado de: https://doi.org/10.46498/reduipb.v24i1.1249

Mendivel, I. (2017). Cultura investigativa y producción científica en la Universidad Nacional de Ingeniería. Recuperado de:http://repositorio.ucv.edu.pe/handle/20.500.12692/43427

MINEDU. (2018). Reforma Universitaria 30220. Recuperado de: http://www.minedu.gob.pe/reformauniversitaria/pdf/ley_universitaria.pdf

MINEDU. (2020a). Ley N²4029 LEY DEL PROFESORADO Texto actualizado con las modificatorias introducidas por la Ley $N^{\circ}$ 25212. Recuperado de: http://www.munisurquillo.gob.pe/municipalizacion-educacion/leyprofesorado-24029.pdf

MINEDU. (2020b). LEY UNIVERSITARIA LEY N²3733. Recuperado de: https://www.albany.edu/dept/eaps/prophe/data/Country_Law/Peru-LEY UNIVERSITARIA_modificada.pdf

MINEDU. (2020c). Norma Técnica: Disposiciones que establecen estándares en progresión de las competencias profesionales del Marco de Buen Desempeño Docente. (p. 43). Recuperado de: https://cdn.www.gob.pe/uploads/document/file/505247/RVM_N_033-2020-MINEDU.pdf

Núñez Rojas, N. (2019). Enseñanza de la competencia investigativa: percepciones y evidencias de los estudiantes universitarios Teaching of research competence: perceptions and evidence of university students Contenido. En ISSN (Vol. 40). Recuperado de: http://www.revistaespacios.com/a19v40n41/a19v40n41p26.pdf

Ponce Lopez, I., Juárez Hernández, L. G., \& Tobón Tobón, S. (2020). Construcción y validación de un instrumento para evaluar el abordaje de la sociedad del conocimiento en docentes. Apuntes Universitarios, 10(1), 40-65. Recuperado de: https://doi.org/10.17162/au.v10i1.417

Quezada Castro, G. A., Castro Arellano, M. del P., Oliva Núñez, J. M., \& Quezada Castro, M. del P. (2020). Autopercepción de la labor docente universitaria: identificando competencias investigativas aplicables en el 2020. Revista Boletín Redipe, 9(1), 164-173. Recuperado de: https://doi.org/10.36260/rbr.v9i1.905 
Ramos Banteurt, A., \& Silva Speck, L. (2019). Two basic conditions for the formation of the discursiveinvestigative competence in professionals of the superior education. Recuperado de: https://search.proquest.com/docview/2245647468/F99C204DEF314986PO/12 ?accountid=37408

Ratte, A., Drees, S., \& Schmidt-Ott, T. (2018). The importance of scientific competencies in German medical curricula - The student perspective. BMC Medical Education, 18(1). Recuperado de: https://doi.org/10.1186/s12909-018-1257-4

Reiban, R., De la Rosa, H., \& Zeballos, J. (2017). Competencias investigativas en la Educación Superior. Competencias investigativas en la Educación Superior Revista Publicando, 4(10), 395-405. Recuperado de: http://scielo.sld.cu/scielo.php?script=sci_arttext\&pid=S2218-36202018000400075

Ricardo Velázquez, M., Amat Abreu, M., Rafael Andrade Santamaría, D., Roberto Jiménez Martínez, M., \& Cinthia Paulina Cisneros Zúñiga, M. (2019). Development of formative research competences: challenges and perspectives for the. Recuperado de: https://search.proquest.com/docview/2245653337/5067EOB71DEF401CPO/12 ?accountid=37408

Rodríguez, L. A., Escobar, M. C., Aveiga, V. I., \& Durán, U. C. (2019). Formative strategy and development of the teaching competence named managing the family educational orientation, in junior high school education. Informacion Tecnologica, 30(6), 277-288. Recuperado de: https://doi.org/10.4067/S071807642019000600277

SUNEDU. (2017). INFORME BIENAL SOBRE LA REALIDAD UNIVERSITARIA PERUANA. Recuperado de: www.sunedu.gob.pe

Tinoco-Cuenca, N. P., Damián-Nuñez, E., Isla-Alcoser, S., \& Bedoya, M. A. M. (2020). Competencias de investigación e impacto socioeducativo en los trabajos de titulación de una universidad de Ecuador. Propósitos y Representaciones, 8(0). Recuperado de: https://doi.org/10.20511/PYR2020.V8N3.442

Turpo-Gebera, O., Quispe, P. M., Paz, L. C., \& Gonzales-Miñán, M. (2020). Formative research at the university: Meanings conferred by faculty at an Education Department. Educacao e Pesquisa, 46, 1-18. Recuperado de:https://doi.org/10.1590/S1678-4634202046215876

Turpo Gebera, O., Gonzales-Miñán, M., Mango Quispe, P., \& Cuadros Paz, L. (2020). Sentidos docentes asignados a la enseñanza de la investigación formativa en una facultad de educación. Propósitos y Representaciones, 8(0). Recuperado de: https://doi.org/10.20511/pyr2020.v8n3.326

UCV. (2020). Guía de Elaboración del Trabajo de Investigación y Tesis para la obtención de Grados Académicos y Títulos Profesionales. Recuperado de:

https://uvcv.edu.pe/pluginfile.php/1349208/mod_resource/content/1/GUIA UCV.pdf

UNESCO. (2020). Docentes. En DOCENTES. Recuperado de: https://es.unesco.org/themes/docentes

Valdés, V., \& Gutiérrez-Esteban, P. (2018). The pedagogical priorities in the learning and knowledge society. An study for the reflection on the quality in the new educational model. Multidisciplinary Journal of Educational Research, 8(1), 1-28. Recuperado de:https://doi.org/10.17583/remie.2018.3199

Vallejo, A. (2020). (2020). El papel del docente universitario en la formación de estudiantes investigadores desde la etapa inicial. scielo.sld.cu. Recuperado de:

http://scielo.sld.cu/scielo.php?script=sci_arttext\&pid=S0864-21412020000200017 
Véliz, A., \& Torres, L. (2017). LAS COMPETENCIAS INVESTIGATIVAS EN DOCENTES. CIENCIA y TECNOLOGÍA, 21(93), 34-42. Recuperado de:https://www.uctunexpo.autanabooks.com/index.php/uct/article/view/147/186

Vezub, L. (2019). Las políticas de Formación Docente Continua en América Latina . Mapeo países. Recuperado de: https://www.buenosaires.iiep.unesco.org/sites/default/files/actividades/2019-06/Vezub\%2C L \%282019\%29 Análisis comparativos de políticas en educación.pdf

Yizza, A. :, Delgado, M., De Vita, N., Trujillo, V., Ramón, R., \& Mendoza, A. (2019). Competencias Investigativas del Docente Construidas durante la Formación Universitaria. Revista Scientific, ISSN-e 2542-2987, Vol. 4, $N^{\circ} .13,2019$ (Ejemplar dedicado a: Revista Scientific), págs. 200-220, 4(13), 200-220. Recuperado de: https://doi.org/10.29394/Scientific.issn.2542-2987.2019.4.13.10.200-220

Alanís Jiménez, J. F. (2018). Consideraciones para la formación continua de personal docente universitario. Revista Educación, 228-249. Recuperado de:https://doi.org/10.15517/revedu.v42i2.25123

Albareda-Tiana, S., Vidal-Raméntol, S., Pujol-Valls, M., \& Fernández-Morilla, M. (2018). Holistic Approaches to Develop Sustainability and Research Competencies in Pre-Service Teacher Training. Sustainability, 10(10), 3698. Recuperado de: https://doi.org/10.3390/su10103698

ANDEAN UNIVERSITY. (2018). Técnicas del Fichaje 2018 -II.doc - Universidad Andina Del Cusco T|u00c9cnicas de estudio y de Investilu00d3n I Actividad inicial de recojo de saberes/ Course Hero. Recuperado de: https://www.coursehero.com/file/42286873/TECNICAS-DEL-FICHAJE-2018-IIdoc/

Baena, G., \& Baena, P. (2017). Metodología de la investigación Grupo Editorial Patria Sistema de aprendizaje en línea Metodología de la investigación. Recuperado de: www.editorialpatria.com.mxwww.sali.org.mx

Banderas Martínez, C., Olivares, G. C., Estela, M., \& Castro, M. (2018). Perspectivas docentes sobre la formación de competencias investigativas en relación con los programas de asignatura. Sincronía, ISSN-e 1562-384X, No. 74 (Julio-Diciembre 2018), 2018, págs. 589-616, 74, 589-616. Recuperado de: https://doi.org/10.32870/sincronia.axxii.n74.29b18

Barbosa-Chacón, Jorge Winston; Jarbosa Herrera, uan Carlos; Rodríguez Villabona, M. (2015). Concepto, enfoque y justificación de la sistematización de experiencias educativas: Una mirada "desde" y "para" el contexto de la formación universitaria. Recuperado de:

http://www.scielo.org.mx/scielo.php?script=sci_arttext\&pid=S0185-26982015000300008

Böttcher, F., \& Thiel, F. (2018). Evaluating research-oriented teaching: a new instrument to assess university students' research competences. Higher Education, 75(1), 91-110. Recuperado de:

https://doi.org/10.1007/s10734-017-0128-y

Buendía Arias, X. P., Zambrano Castillo, L. C., \& Insuasty, E. A. (2017). El desarrollo de competencias investigativas de los docentes en formación en el contexto de la práctica pedagógica. Revista Folios, 47. Recuperado de: https://doi.org/10.17227/folios.47-7405

Castro Taks, A. (2020). ELABORACIÓN DE FICHAS ¿Qué son las fichas? Recuperado de: https://investigar1.files.wordpress.com/2010/05/elaboracic3b3n-de-fichas.pdf

CEGOS. (2020). La importancia de la formación continua para el profesional - Cegos. Recuperado de: https://www.cegosonlineuniversity.com/la-importancia-de-la-formacion-continua-para-el-profesional/ 
Contreras, F. A. G., \& Maluk Uriguen, S. A. (2017). A descriptive analysis of university administration in ecuador from the perspective of legislative change. Revista Electronica de Investigacion Educativa, 19(2), 22-37. Recuperado de: https://doi.org/10.24320/redie.2017.19.2.866

D’olivares Durán, N., Liliana, C., \& Cifuentes, C. (2019). Competencias investigativas: inicio de formación de jóvenes investigadores en educación media. Humanismo. Soc, 7(1), 2019. Recuperado de: https://doi.org/10.22209/rhs.v7n1a01

Damián Cabezas Mejía, E., \& Andrade Naranjo Johana Torres Santamaría, D. (2018). Introducción a la metodología de la investigación científica. Recuperado de: www.repositorio.espe.edu.ec.

De Cos Gonzalez-Taladriz, Lucía \& Brizo-Montiano, S. M. (2017). Mendeley Guía de uso Grupo de Trabajo de Gestores Bibliográficos. Recuperado de: https://biblioteca.ucm.es/data/cont/docs/382-2018-01-23Manual Mendeley 3 ed. (Noviembre 2016)reducido.pdf

De los Santos Alejos, M. A. (2015). El fichaje como metodo de investigacion. Recuperado de: https://es.slideshare.net/MADLSA/el-fichaje-como-metodo-de-investigacion

Delgado, et al. (2019). Competencias Investigativas del Docente Construidas durante la Formación Universitaria. Revista Scientific, 4(13), 200-220. Recuperado de: https://doi.org/10.29394/scientific.issn.2542-2987.2019.4.13.10.200-220

Díaz de Salas, Y. del C. (2020). Mirada Fenomenológica desde las Competencias Investigativas en las Líneas de Investigación de la UNESR. Revista Scientific, 5(15), 269-287. https://doi.org/10.29394/scientific.issn.2542-2987.2020.5.15.13.269-287

Dios, I., Calmaestra, J., \& Rodríguez-Hidalgo, A. J. (2018). Validación de la escala de competencias docentes organizacionales y didácticas para educadores. Revista Mexicana de Investigacion Educativa, 23(76), 281302.

Expósito Unday, Dámari ; Gonzales Valero, J. A. (2017). Sistematización de experiencias como método de investigación. http://scielo.sld.cu/scielo.php?script=sci_arttext\&pid=S1608-89212017000200003

Feixas, M., \& Zellweger, F. (2019). Formación e innovación docente universitaria para una educación transformadora: la investigación e indagación reflexiva como requisito. El Guiniguada. Revista de investigaciones y experiencias en Ciencias de la Educación. https://doi.org/10.20420/elguiniguada.2019.265

Flores Nessi, E. M., Loaiza Falcón, A. C., \& Rojas de Ricardo, G. N. (2020). Rol del docente investigador desde su práctica social. Revista Scientific, 5(15), 106-128. https://doi.org/10.29394/scientific.issn.25422987.2020.5.15.5.106-128

Gallegos, M. C., Peralta, C. A., \& Guerrero, W. M. (2017). Utilidad de los gestores bibliográficos en la organización de la información para fines investigativos. Formacion Universitaria, 10(5), 77-85. https://doi.org/10.4067/S0718-50062017000500009

Gómez-Escorcha, J. A., Isea Araque, N., \& López Lara, V. de las M. (2019). Competencias investigativas y su influencia en la formulación de proyectos de investigación en la Universidad Valle del Momboy. Actualidades Pedagógicas, 1(73), 191-213. https://doi.org/10.19052/ap.vol1.iss74.10 
Gómez-Luna, E., Fernando-Navas, D., Aponte-Mayor, G., \& Betancourt-Buitrago, L. A. (2014). Metodología para la revisión bibliográfica y la gestión de información de temas científicos, a través de su estructuración y sistematización. DYNA (Colombia), 81(184), 158-163. https://doi.org/10.15446/dyna.v81n184.37066

Goris, G. (2015). UTILIDAD Y TIPOS DE REVISIÓN BIBLIOGRÁFICA / Guirao Goris / Revista Ene de Enfermería. http://ene-enfermeria.org/ojs/index.php/ENE/article/view/495/guirao

Guamán Gómez, Verónica Jacqueline, Herrera Martínez, Lázara, \& Espinoza Freire, E. E. (2020). Las competencias investigativas como imperativo para la formación de conocimientos en la universidad actual. http://scielo.sld.cu/scielo.php?script=sci_arttext\&pid=S1990$86442020000100083 \& \operatorname{lng}=e s \& n r m=i s o \&$ tIng=es

Guía Normas APA $7^{a}$ edición. (2020). https://normas-apa.org/

Hubbard, K. E., \& Dunbar, S. D. (2017). Perceptions of scientific research literature and strategies for reading papers depend on academic career stage. PLOS ONE, 12(12). https://doi.org/10.1371/journal.pone.0189753

Hüseyin Şahan, H., \& Tarhan, R. (2015). International Journal of Psychology and Educational Studies Scientific Research Competencies of Prospective Teachers and their Attitudes toward Scientific Research ARTICLE INFO ABSTRACT. International Journal of Psychology and Educational Studies, 2(3), 20-31. https://doi.org/10.17220/ijpes.2015.03.003

Lopéz- de Parra, L., Polanco-Perdomo, V., \& Correa-Cruz, L. (2017). Mirada a las investigaciones sobre formación investigativa en la universidad latinoamericana: estado del arte 2010 a 2017. REVISTA DE INVESTIGACIÓN, DESARROLLO E INNOVACIÓN, 8(1), 77. https://doi.org/10.19053/20278306.v8.n1.2017.7371

López Carreño, M. (2015). Análisis comparativo de los gestores bibliográficos sociales Zotero, Docear y Mendeley: características y prestaciones.

https://digitum.um.es/digitum/bitstream/10201/51054/1/Analisis comparativo de los gestores bibliograficos sociales Zotero, Docear y Mendeley caracteristicas y prestaciones..pdf

Luna-menecio, A. A. (2020). La construcción de competencias investigativas del docente de posgrado para lograr el desarrollo social sostenible. https://www.revistaespacios.com

Matute, L. J., \& Pereira Rodríguez, Z. (2020). Práctica investigativa: Diferentes tiempos y visiones en la formación universitaria. Revista EDUCARE - UPEL-IPB - Segunda Nueva Etapa 2.0, 24(1), 303-319. https://doi.org/10.46498/reduipb.v24i1.1249

Mendivel, I. (2017). Cultura investigativa y producción científica en la Universidad Nacional de Ingeniería. http://repositorio.ucv.edu.pe/handle/20.500.12692/43427

MINEDU. (2018). Reforma Universitaria 30220. http://www.minedu.gob.pe/reformauniversitaria/pdf/ley_universitaria.pdf

MINEDU. (2020a). Ley N²4029 LEY DEL PROFESORADO Texto actualizado con las modificatorias introducidas por la Ley $N^{\circ}$ 25212. http://www.munisurquillo.gob.pe/municipalizacion-educacion/ley-profesorado24029.pdf

MINEDU. (2020b). LEY UNIVERSITARIA LEY N² 23733. https://www.albany.edu/dept/eaps/prophe/data/Country_Law/Peru-LEY UNIVERSITARIA_modificada.pdf 
MINEDU. (2020c). Norma Técnica: Disposiciones que establecen estándares en progresión de las competencias profesionales del Marco de Buen Desempeño Docente. (p. 43). https://cdn.www.gob.pe/uploads/document/file/505247/RVM_N_033-2020-MINEDU.pdf

Núñez Rojas, N. (2019). Enseñanza de la competencia investigativa: percepciones y evidencias de los estudiantes universitarios Teaching of research competence: perceptions and evidence of university students Contenido. En ISSN (Vol. 40). http://www.revistaespacios.com/a19v40n41/a19v40n41p26.pdf

Ponce Lopez, I., Juárez Hernández, L. G., \& Tobón Tobón, S. (2020). Construcción y validación de un instrumento para evaluar el abordaje de la sociedad del conocimiento en docentes. Apuntes Universitarios, 10(1), 40-65. https://doi.org/10.17162/au.v10i1.417

Quezada Castro, G. A., Castro Arellano, M. del P., Oliva Núñez, J. M., \& Quezada Castro, M. del P. (2020). Autopercepción de la labor docente universitaria: identificando competencias investigativas aplicables en el 2020. Revista Boletín Redipe, 9(1), 164-173. https://doi.org/10.36260/rbr.v9i1.905

Ramos Banteurt, A., \& Silva Speck, L. (2019). Two basic conditions for the formation of the discursiveinvestigative competence in professionals of the superior education. https://search.proquest.com/docview/2245647468/F99C204DEF314986PQ/12 ?accountid=37408

Ratte, A., Drees, S., \& Schmidt-Ott, T. (2018). The importance of scientific competencies in German medical curricula - The student perspective. BMC Medical Education, 18(1). https://doi.org/10.1186/s12909-0181257-4

Reiban, R., De la Rosa, H., \& Zeballos, J. (2017). Competencias investigativas en la Educación Superior. Competencias investigativas en la Educación Superior Revista Publicando, 4(10), 395-405. http://scielo.sld.cu/scielo.php?script=sci_arttext\&pid=S2218-36202018000400075

Ricardo Velázquez, M., Amat Abreu, M., Rafael Andrade Santamaría, D., Roberto Jiménez Martínez, M., \& Cinthia Paulina Cisneros Zúñiga, M. (2019). Development of formative research competences: challenges and perspectives for the. https://search.proquest.com/docview/2245653337/5067EOB71DEF401CPO/12?accountid=37408

Rodríguez, L. A., Escobar, M. C., Aveiga, V. I., \& Durán, U. C. (2019). Formative strategy and development of the teaching competence named managing the family educational orientation, in junior high school education. Informacion Tecnologica, 30(6), 277-288. https://doi.org/10.4067/S0718-07642019000600277

SUNEDU. (2017). INFORME BIENAL SOBRE LA REALIDAD UNIVERSITARIA PERUANA. www.sunedu.gob.pe

Tinoco-Cuenca, N. P., Damián-Nuñez, E., Isla-Alcoser, S., \& Bedoya, M. A. M. (2020). Competencias de investigación e impacto socioeducativo en los trabajos de titulación de una universidad de Ecuador. Propósitos y Representaciones, 8(0). https://doi.org/10.20511/PYR2020.V8N3.442

Turpo-Gebera, O., Quispe, P. M., Paz, L. C., \& Gonzales-Miñán, M. (2020). Formative research at the university: Meanings conferred by faculty at an Education Department. Educacao e Pesquisa, 46, 1-18. https://doi.org/10.1590/S1678-4634202046215876

Turpo Gebera, O., Gonzales-Miñán, M., Mango Quispe, P., \& Cuadros Paz, L. (2020). Sentidos docentes asignados a la enseñanza de la investigación formativa en una facultad de educación. Propósitos y Representaciones, 8(0). https://doi.org/10.20511/pyr2020.v8n3.326 
UCV. (2020). Guía de Elaboración del Trabajo de Investigación y Tesis para la obtención de Grados Académicos y Títulos Profesionales. https://uvcv.edu.pe/pluginfile.php/1349208/mod_resource/content/1/GUIA UCV.pdf

UNESCO. (2020). Docentes. En DOCENTES. https://es.unesco.org/themes/docentes

Valdés, V., \& Gutiérrez-Esteban, P. (2018). The pedagogical priorities in the learning and knowledge society. An study for the reflection on the quality in the new educational model. Multidisciplinary Journal of Educational Research, 8(1), 1-28. https://doi.org/10.17583/remie.2018.3199

Vallejo, A. (2020). (2020). El papel del docente universitario en la formación de estudiantes investigadores desde la etapa inicial. scielo.sld.cu. http://scielo.sld.cu/scielo.php?script=sci_arttext\&pid=S086421412020000200017

Véliz, A., \& Torres, L. (2017). LAS COMPETENCIAS INVESTIGATIVAS EN DOCENTES. CIENCIA y TECNOLOGÍA, 21(93), 34-42. https://www.uctunexpo.autanabooks.com/index.php/uct/article/view/147/186

Vezub, L. (2019). Las políticas de Formación Docente Continua en América Latina . Mapeo países. https://www.buenosaires.iiep.unesco.org/sites/default/files/actividades/2019-06/Vezub\%2C L \%282019\%29 Análisis comparativos de políticas en educación.pdf

Yizza, A. :, Delgado, M., De Vita, N., Trujillo, V., Ramón, R., \& Mendoza, A. (2019). Competencias Investigativas del Docente Construidas durante la Formación Universitaria. Revista Scientific, ISSN-e 2542-2987, Vol. 4, $N^{0}$. 13, 2019 (Ejemplar dedicado a: Revista Scientific), págs. 200-220, 4(13), 200-220. https://doi.org/10.29394/Scientific.issn.2542-2987.2019.4.13.10.200-220

\section{Anexos}

\section{Cuadro 1}

Modelo de ficha sincrética

\begin{tabular}{|l|l|}
\hline $\begin{array}{l}\text { 5. Capítulo de } \\
\text { ubicación en el } \\
\text { trabajo }\end{array}$ & $\begin{array}{l}\text { 1. Título del trabajo científico } \\
\text { 2. Fuente de referencia }\end{array}$ \\
\hline $\begin{array}{l}\text { 6. Número de } \\
\text { página en el } \\
\text { trabajo }\end{array}$ & 3. Tema y desrrollo del contenido con la información obtenida \\
\hline
\end{tabular}

Fuente: Elaboración propia 